\title{
Realizing autonomy in responsive relationships
}

\author{
Albine Moser - Rob Houtepen - Cor Spreeuwenberg • \\ Guy Widdershoven
}

Published online: 27 March 2010

(C) The Author(s) 2010. This article is published with open access at Springerlink.com

\begin{abstract}
The goal of this article is to augment the ethical discussion among nurses with the findings from empirical research on autonomy of older adults with type 2 diabetes mellitus. There are many factors influencing autonomy. These include: health conditions, treatment, knowledge, experience and skills, personal approach as well as familial patterns, type of relationship, life history and social context. Fifteen older adults with type 2 diabetes mellitus were interviewed in a nurse-led diabetes clinic. These participants perceive three processes which support autonomy in responsive relationships: preserving patterns of concern and interaction, nurturing collaborative responsibilities and being closely engaged in trustful and helpful family relations. People with diabetes realize autonomy in various
\end{abstract}

\footnotetext{
A. Moser $(\bowtie)$

Department of General Practice, Faculty of Health, Medicine and Life Science, Care and Public Health Research Institute, Maastricht University, P.O. Box 616, 6200 MD Maastricht, The Netherlands

e-mail: albine.moser@hag.unimaas.nl
}

A. Moser

Faculty of Health and Care, Zuyd University, Heerlen, The Netherlands

\section{R. Houtepen}

Department of Health, Ethics and Society, Care and Public Health Research Institute, Maastricht University, Maastricht, The Netherlands

C. Spreeuwenberg

Care and Public Health Research Institute, Maastricht

University, Maastricht, The Netherlands

G. Widdershoven

Department of Medical Humanities, EMGO Institute for Health and Care Research, VU University Medical Center, Amsterdam, The Netherlands responsive relationships in their unique life context. Next, we performed a literature review of care ethics and caring in nursing with regard to relational autonomy. We classified the literature in five strands of care: attitude-oriented, dialogue-oriented, activity-oriented, relationship-oriented and life-oriented. According to our respondents, autonomy in responsive relationships is fostered when patient, nurses, professionals of the health team and family members carry out care activities supported by a relational attitude of care. They can best realize autonomy in relationships with others when several essential aspects of care and caring are present in their lives. Therefore, we advocate a comprehensive approach to care and caring.

Keywords Caring activity $\cdot$ Caring attitude $\cdot$ Care ethics . Nurse-led clinic · Patient's perspective .

Relational autonomy $\cdot$ Responsive relationships .

Type 2 diabetes mellitus

\section{Introduction}

Chronic illness such as diabetes may change peoples' lives dramatically. Living with diabetes means having to care for one's health in a situation of threatened autonomy. People with diabetes have to negotiate autonomy in their daily activities (Ingadottir and Halldorsdottir 2008) and in their relationships with professionals and family (Williams and Wood 1988). They need collaborative relationships and support (Mamhidir and Lundman 2004; Nagelkerk et al. 2006; Paterson et al. 1998; Paterson and Sloan 1994). This paper focuses on autonomy within responsive relationships between older adults with diabetes and diabetes specialist nurses (DSN), health professionals and family. The goal is to contribute to the theoretical and ethical discussion using 
findings from empirical research on the autonomy of older adults with type 2 diabetes mellitus. First, we will introduce the findings of a larger grounded theory study, on which our interpretation of autonomy in responsive relationships is based. Next, we present our empirical findings in which we portray how older adults with type 2 diabetes realize autonomy in responsive relationships with others. Then, we will describe five strands of care and caring based on a literature overview. Finally, we discuss our findings in the light of the literature reviewed.

\section{The research project}

This study is part of a larger research project regarding the autonomy of older people with type 2 diabetes mellitus in a nurse-led clinic (Moser et al. 2009, 2006, 2008a, b). The purpose of this project is to identify what issues need particular attention to support patient autonomy in diabetes care, provided by DSN and to formulate recommendations to promote patient autonomy on the individual and policy level. A finding from earlier research is that older adults with type 2 diabetes perceive autonomy as 'competency in shaping one's life' (Moser et al. 2006). Competency is the individual repertoire of skills that includes recognizing possibilities and having the abilities, capacities, and expertise that enable people with diabetes to shape their own lives. Older adults with diabetes initiate and complete various actions daily, which are conceptualized as dimensions of autonomy. To shape one's life with diabetes means that a person with diabetes actively strives towards an autonomy that is right for this particular person in a particular situation; it is based on characteristics that are unique to the person, and it is flexible in changing health conditions and life situations. Shaping one's life is a construct combining several dimensions. The synthesis of diverse dimensions arises in various ways. There are many factors influencing autonomy and the contexts in which shaping one's life occurs. These include: health conditions, treatment, knowledge, experience and skills, personal approach as well as familial patterns, type of relationship, life history and social context. Thus, the combination of the dimensions of autonomy is not fixed, but rather a mix of what is most appropriate at a given situation.

In this article we focus on autonomy in responsive relationships (Moser et al. 2006) which evolves through social interactions and includes reacting to each other's thoughts, feelings, concerns, and habits. Certain behavioural rituals or patterns emerge from these interactions. Verbal and non-verbal cues flow freely between people with diabetes and their nurses, other professionals and their family caregivers which help guide decisions and activities, resulting in outcomes that suit the situation and relationships best. Decisions are made both explicitly and implicitly as the relationship unfolds, for example when it becomes long-term. Responsibilities towards each other are presumed in the sense that care participants know what (care) responsibilities they can expect from each other (Moser et al. 2006).

\section{Method}

To explore the process of autonomy in responsive relationships as described by older adults with type 2 diabetes mellitus we undertook an exploratory, qualitative study. In line with symbolic interactionism (Blumer 1969), we studied people's social interactions and the process of meaningmaking with regard to autonomy in responsive relationships with others. We also used the grounded theory method (Strauss and Corbin 1990) which is an inductive, fromthe-ground-up approach, that uses everyday behaviours to generate a theory about a particular phenomenon (Strauss and Corbin 1990). Grounded theory provides a way to go beyond experience - to move it from description of what is happening to understanding the process by which it is happening (Artinian 1998).

All 15 participants in this study were Dutch and lived in or around the city of Maastricht. These older adults had a confirmed diagnosis of type 2 diabetes and a stable but complex health condition. They were living independently at home, were enrolled for at least 1 year at the nurse-led shared-care unit, were capable of completing an interview of about $1.5 \mathrm{~h}$, and without cognitive impairment or severe geriatric symptoms (diagnosed by a physician). Newly diagnosed patients, Type 2 diabetes patients living in assisted-living housing arrangements or with cognitive impairment and severe geriatric symptoms (diagnosed by a physician), and elderly type 1 diabetic patients were excluded.

The DSN sent people with type 2 diabetes an information letter that included information about the research and asked consent for forwarding their personal data to the researcher. After sending this letter, the DSN contacted the patients to ask whether these data could, indeed, be given to the researcher. Then, the researcher approached these people to see if they were willing to participate and to make interview appointments. All those contacted by the researcher agreed to participate. The participants gave written informed consent prior to the interviews. The respondents were assured that interview data would be dealt with confidentially. Anonymity was secured by codenumbering the interviews. The Ethics Commission of University Hospital Maastricht and Maastricht University gave ethical approval. 
Fifteen in-depth interviews took place and all participants were interviewed at home so that the environment was comfortable and familiar to them. The interview guide consisted of open-ended questions directed towards aspects of autonomy related to daily life in its broadest sense, like "How would you describe your visit to the nurse?" "How is your spouse involved in the care of your diabetes?" Participants were encouraged to provide examples to support their statements and researchers asked follow-up questions. For the married participants, questions regarding the spouse's involvement were: "What were his/her tasks?" or "Who took the initiative to deal with things in that way?" The interviews were tape-recorded and transcribed verbatim. Field notes were taken of observations and impressions during the interview and of the informal talk after each interview.

Using a grounded theory approach, theory was developed inductively from the interview data and deductively through a constant comparative method (Strauss and Corbin 1990). We broke the data into smaller parts. Every line was coded using open codes that were often descriptions used by the participants. We made as many codes as possible to ensure broad coverage. Next, we compared and contrasted the incidences and codes and grouped them into categories. We made sure that categories included similar incidents and codes, yielding increasingly complex and inclusive categories. The phenomenon represented by the category was given a conceptual name. Subsequently, we employed axial coding. We put the data back together in new ways by making connections between the categories and sub-categories. Next, we used selective coding to select the core category and related it to the other categories. Finally, we integrated the data to form a substantive theory. Theoretical sampling (Strauss and Corbin 1990) involved asking specific questions about the emerging codes and categories in subsequent interviews with new respondents. In the first interviews we asked general questions, and later, more focused ones to strengthen our theory. Memos were written throughout the analysis process. After analysis of 12 interviews, saturation occurred which means that data were collected to a point where a sense of closure was attained because new data provided redundant theoretical information. The remaining 3 interviews were used to be sure that no new categories emerged. The analysis resulted in a substantive theory of patient autonomy. A substantive theory evolves from the study of a phenomenon situated in one particular context (Strauss and Corbin 1990).

\section{Findings}

People with type 2 diabetes mellitus identify three processes which support autonomy in responsive relationships: preserving patterns of concern and interaction, nurturing collaborative responsibilities, and being closely involved in trusting and helpful relations. The first two processes relate to the health care context and the latter to the family environment.

\section{Preserving patterns of concern and interaction}

The nurse-patient relationship is a responsive relationship which develops over time. Both parties are engaged and give shape to this process. These older adults with diabetes feel the concern which DSN bring to their healthcare. A certain mode of interaction develops and responses to these interactions steer that relationship. Responses can be verbal as well as non-verbal, like eye contact or nodding to acknowledge a patient's experiences, or physical, such as the performance of care activities. The DSN and the person with diabetes agree, explicitly or implicitly, on care and treatment requirements, in terms of routines and habits, by communicating or interacting with each other. The nurse and the patient then know each other's responsibilities and requirements and act accordingly.

The visit is always the same. We have found a rhythm. If things are fine it does not change. She asks: How are you? Have there been any noteworthy things since the last visit? Then we check the blood results and sugar profiles. Once a year she checks on my feet. She also asks: When will you see the ophthalmologist? Then I tell her when I have scheduled the appointment. Since I know that she asks about the ophthalmologist, I make regular appointments. She writes it down in my file. She is really concerned about the care of my diabetes. But I also do my best to make sure that things are as well as can be. We have become a good team.

\section{Nurturing collaborative responsibilities}

People with diabetes also develop a responsive relationship with other health professionals who have a stake in their care. In the nurse-led clinics, various healthcare professionals from different disciplines and settings work together as a team based on a distinct set of responsibilities. In daily practice, care responsibilities are a coordinated series of (care) activities that are defined and agreed upon among the diabetes team members. The care process, thus, involves many health professionals that are part of the diabetes team. The number of members of the health team of individual patients is not static. Depending on the health of the person with diabetes, different professionals are involved at different times. If a person with diabetes needs additional care, such as dietary advice, for example, the 
dietician becomes a member of the diabetes team, sometimes permanently and sometimes temporarily. In this way, people with diabetes receive comprehensive care from the members of the health team whether in the form of direct or indirect care activities. Patients and health professionals collaborate and take on care responsibilities as appropriate. This is possible in the nurse-led clinic because the various professionals value and appreciate each other's contributions to the team effort, each with their own professional and relational responsibilities with regard to the patient and other members of the health team.

My nurse discusses my health and treatment with my GP. Once, while I was at the GP I asked if they could check my blood pressure. Next, the doctor's assistant also checked my blood sugar level. That day my sugar level was high. The day after [name of diabetes specialist nurse] called and said: I got a phone call... I think it is very exceptional that the assistant of my GP contacted [name of diabetes specialist nurse] and that she got back to me. This is special. I appreciate that.

Being engaged in trustful and helpful family relations

Responsive relationships with family members differ from those with professionals. In the family-patient relationship a responsive relationship has developed over many years. In such a relationship, the person with diabetes and the family member, usually the spouse know each other well and trust each other. Both are engaged with each others' concerns. The amount of involvement depends on the given situation and what family members consider appropriate within the responsive relationship. In some cases spouses are not too involved while in other cases or circumstances spouses are very closely engaged. It varies accordingly and in any case, family members assume responsibilities.

He [husband] is very closely engaged. We have always been very close and we do everything together. He always helps me. In the morning I get up, I inject insulin, I prepare breakfast and then I take my medication. This is my part. During the day we do nice things together like visiting our grandchildren. In the evening he assumes responsibilities. He cooks and when he is ready he says: The meal is almost done. I inject insulin and then we have dinner.

The family context is full of relational patterns in which the issue of autonomy is inherent. The extent to which responsibility is assumed by the patient and family member is the extent to which the person with diabetes is autonomous in that relationship. Often spouses deal with diabetes care issues as they deal with other care issues within the family context. This seems natural for spouses who have known each other for a long time and share a common past. In most cases diabetes care responsibilities and tasks are integrated seamlessly into these existing responsive family relationships and are embedded in the daily healthcare routine, accordingly. Occasionally there are struggles, for example when a family member is kind of overprotective. Then this issue is solved in the way any other problem is handled within that relationship.

\section{Literature overview}

In recent years, there is a growing interest in the relational aspects of autonomy, usually associated with care ethics. Gilligan (1982) was a pioneer of an ethic of care and responsibility which centres on the moral understanding of responsibilities and relationships. Receptivity, relatedness and responsiveness (Noddings 1984) are at the core. In past decades this stance has taken on several forms. The departure point for our literature overview was to find publications that related to autonomy as realized in responsive relationships. We started with literature in the field of care ethics. Then, we extended our review to nursing with caring as the central focus. Next, we included literature beyond the field of ethics and caring that highlighted relational aspects. We classified the literature into five relational contexts in which caring takes place: attitude-oriented, dialogue-oriented, activity-oriented, relationship-oriented and life-oriented. Due to the overlap and interrelatedness of the theories we were careful to categorize this heterogeneous sample according to the major theoretical focus of the authors. For example, Gastmans (1999) relates caring attitudes of nurses to life as a whole. We could have classified this theory as life-oriented. However, since he primarily emphasizes the nurse's attitude we chose to place his approach in the attitude-oriented strand. In this way we identified the essential focus of the authors and at the same time highlighted the diversity of these theories.

An attitude-oriented care ethic views care as a personal quality or virtue. Verkerk (2001) highlights attitudes such as commitment and attentiveness to the needs of the patients. Nurses should take an active and committed role following and directing patients when necessary. Hagerty and Patusky (2003) describe four qualities that are essential to establish relatedness in relationships: sense of belonging, reciprocity, mutuality and synchrony. Gastmans (1999) defines caring as a moral attitude, as a sensitive and supportive response of a nurse to the situation and circumstances of a vulnerable human being who is in need of help. He links the characteristics of a good nurse to the concepts of a good person and a good life. Responsible nurses 
develop a caring attitude throughout their life which is expressed in a unique way by each individual given their personality traits (Gastmans 1999). In comparison, Verkerk (2001) and Hagerty and Patusky (2003) mention attitudes only in relation to the profession of nursing. Nurses support patient autonomy by exhibiting and cultivating such traits and attitudes as commitment and reciprocity.

Whereas attitude-oriented theories focus on the nurses' individual characteristics, dialogue-oriented theories center on the communicative processes among those who belong to the patients' network. Some conceptualize dialogical autonomy in the care (Widdershoven 1999) and nursing (Krouse and Roberts 1989; Roberts et al. 1995) context. Widdershoven (1999) defines dialogical autonomy as participating in care, including decision-making, while Krouse and Roberts (1989) define it as a final agreement in a decision made by agreement from the care-receiver and the nurse. In the family-patient relationship the autonomy of all family members should be respected by means of family dialogue (Donchin 2000) or family conferences (Kuczewski 1996). This provides opportunities for all participants to articulate their perspectives, air disagreements, and empathically perceive others' viewpoints (Donchin 2000). Donchin (2000) points out that in the process of realizing autonomy the patients' wishes need to take precedence, while Kuczewski (1996) claims that all family members who are involved in the patient's care should participate in the dialogue. Hardwig (1990) argues that family members assist the patients in interpreting their values and translating them into treatment preferences. Autonomous patients actively take part in dialogue concerning care decisions in the care, nursing and family contexts.

While dialogue-oriented theories focus on communication processes, an activity-oriented strand emphasizes the activity of care itself in interactions with others. According to Struhkamp (2005) care happens in the day-to-day work in institutional settings and in the interaction with professional and non-professional care-givers. For Mol (2006), care means actorship. Professional actorship happens in the medical environment and patients take on actorship in daily life. Actorship is fluid and reciprocal since taking care of and being cared for alternate. The actors' roles are interdependent. Professionals cannot make an effective treatment plan if patients do not carry out the appropriate care activities. Acting means one provides care, tries out different treatments, tinkers, adjusts, with an uncertain outcome. In this sense, care as an activity is a continuous process. Struhkamp (2005) claims that autonomy is realized through care activities to support the continuity of the biography and authentic life-style of patients at many locations, while Mol (2006) concentrates on the consultation room. The main point to be drawn from these is that the process of patient autonomy takes shape by continuously carrying out certain care activities in an interactive manner in several contexts.

The relationship-oriented strand differs from the previous one because the relationship is not solely based on the activity of care but is characterized by being engaged with one another. At this point we focus on the nurse-patient relationship. Parker (1990) describes a nurse-patient relationship as the co-construction of meaning between nurses and patients. As the journey unfolds, the nurse and patient are mutually receptive to change, and both are aware that the final destination remains open to negotiation. According to Tarlier (2004) a caring nurse-patient relationship encompasses three elements: respect, trust and mutuality. Mutuality is a negotiation process between the nurse and patient as the relationship develops. Tarlier (2004) relates negotiation to the construction of the relationship, whilst Parker (1990) considers negotiation to be the final outcome. Peplau (1988) conceptualizes the nurse-patient relationship as a process to solve health problems: at one end are two people with separate goals and interests, and at the other end, two people working together to solve a health difficulty about which there is a common understanding. According to Watson (1988) a transpersonal caring relationship begins when nurses enter into the phenomenal field of patients, are able to detect the patients' state of being, empathize, and respond to the condition in such a way that it evokes the patients' feelings and thoughts. Watson (1988) puts the patient into a receiving role. This is in contrast to the other theorists who focus on negotiation and common understanding. The essence is that patients are involved in and give shape to their relationships with nurses. These relationships encompass attitudes, dialogue and activities.

The relationship-oriented strand focuses on the nursepatient relationship whereas the life-oriented strand refers to the context in which people live. Tronto (1993) defines care in its broadest sense, as activities that include everything we do to maintain, continue and repair our world. Care is an ongoing process in a certain context which includes the micro, meso and macro level as well as the private and public sphere. The caring process consists of four phases: caring about, taking care of, care-giving and care-receiving. From these four process phases, four ethical qualities arise which make care a moral practice: attentiveness, responsibility, competence and responsiveness. Thus, care is both a practice and a disposition. While Tronto focuses on care in the broadest sense, Friedemann and Köhlen (2003) are more specific and relate it to the family context. The theory of family and environmental nursing (Friedemann and Köhlen 2003) describes three levels of family: individual family members, the interpersonal level and the family system level. The last one includes structural and functional system components 
interacting with the broader environment which influence family systems. The goal of all interventions are family system change (Friedemann 1989). In family nursing, care is not delivered but actively shaped together with the family system. Nursing care starts with building relationships with the members of the family system and nursing goals are shared (Friedemann 1989). In this strand, the essence of realizing autonomy is that patients are part of the (nursing) care process. In sum, these strands provide various perspectives on the process through which patients can realize autonomy in responsive relationships.

\section{Discussion}

We investigated how older adults with type 2 diabetes mellitus perceive autonomy in responsive relationships with others. Autonomy is maintained and fostered in supportive relationships. This process is dynamic and may vary from situation to situation and relationship to relationship. The result is not necessarily an optimum, since there are no independent criteria for such a qualification. Given the responsive nature of the processes, including certain give-and-take aspects, the results can usually be qualified as a sort of compromise.

Three kinds of responsive relationships are highlighted in this study: the person with diabetes with DSN, other professionals in the healthcare team and the family. Nurse theorists (Peplau 1988; Tarlier 2004; Watson 1988) primarily focus on the nurse-patient relationship since caring is seen as the essence of nursing as expressed in the nursepatient relationship. They consider the broader patient context as relevant but do not specify it. This is surprising since some nurse scholars claim that nurses pursue "holistic" caring (Friedemann 1989; Friedemann and Köhlen 2003; Watson 1988). Exceptions are Friedemann and Köhlen (2003) who state that family nursing is an integral part of all fields of nursing. They also consider the broader environment, such as the community in which a family lives, to be important in family system nursing care. Philosophers have already acknowledged that relational autonomy develops in different contexts. Some (Struhkamp 2005; Widdershoven 1999) relate it to the context of health care institutions and the patients' private environment. Gastmans (1999) links caring attitudes of nurses to life as a whole and Tronto (1993) relates caring to everything people do. According to our findings, people with diabetes have a unique life context and unique responsive relationships within which they realize autonomy.

As our second segment shows, patients exercise autonomy in responsive relationships by nurturing collaborative responsibilities with the health team. The organization of chronic care has become increasingly specialized and fragmented: a number of professionals work together to care for one chronically ill patient. Organizational arrangements are important to realize autonomy in relations with others (Struhkamp 2005; Tronto 1993). According to Mol (2006), care has no defined boundaries, it is an open process that takes place in a care team with a variety of tasks. Patients are also participants in the care team. She underscores that it is important to clarify what task is done by whom. Verkerk (2001) is more specific about the division of tasks: the nurse's responsibility is to be attentive to the needs of the patients and patients should be responsive to the care received. Tronto (1993) provides the most comprehensive view of the division of tasks. She differentiates between four phases. In the first three phases, it is the professionals and informal care providers who take on responsibilities. The last phase focuses on care-receiving, the patient's response to the care received. Tronto (1993) points out that these phases may occur at different levels of the health care system and that the care-giving process is only complete if all phases are well integrated into a whole. When this is the case, then people with diabetes receive autonomy in nurturing collaborative responsibilities.

As is clear from our third segment, patient autonomy in responsive relationships is not necessarily dialogue-oriented, although conversation and dialogue are part of it (Widdershoven 1999). In the family context the extent to which autonomy is realized is influenced by the particular pattern of interaction. It is less overt in communication processes but more apparent in care activities that typify the specific relationship. In general, dialogical care ethics tends to give too little attention to the nature of the care work being given although some (Widdershoven 1999) advocate a broader notion of dialogue. Family-care-giving is becoming increasingly important since more and more people with chronic conditions will rely on the help of family members. If nurses want to provide good care, they should take on the challenge to facilitate responsive relationships in families. Ideally, nurse scholars should move away from nurse-focused theories and towards patientcentered theories (Morse et al. 1990) in which family and the patient's life context become additional facets of the nurse-patient relationship. By definition, family relationships precede relationships with professional caregivers and are, thus, a natural context wherein the patient exercises autonomy.

There are four elements that older adults with diabetes consider as important resources: the distribution of responsibilities, relational patterns, character traits and care activities. The realization of autonomy relies on situational factors at any given time, for example, health situation or context. Furthermore, due to the dynamic nature of a patient's situation, the responsibilities of patients, professionals and family members may be redefined and changed 
accordingly. Autonomy in responsive relationships brings into play those parts of care that do not centre on decisions but are vital to the patients' exercise of autonomy. Autonomy is broader than exercising authority in decisionmaking situations (Struhkamp 2005) and it is not necessarily an explicit agenda item. In contrast, our findings show that autonomy is embedded in relational pattern and is subtle, implicit, less conscious and less directive. In our study, older adults with diabetes also draw on caring traits and care activities. For example, we interpret preserving patterns of concern and interactions as a combination of character traits (preserving patterns of concern) and care activities (preserving patterns of interaction). Both are interconnected and necessary to realize autonomy. A trait is an attitude to sustain a relationship with an older adult with diabetes. A care activity is an action related to diabetes care. The action might be practical, such as performing the actual care intervention, but it also includes actions such as having conversations or listening. Depending on the context of the responsive relationship, a specific trait is at the foreground and that trait influences care activities that need to be carried out. Caring actions have an ethical value in light of the quality of the caring attitude (Gastmans 1999). Tronto (1993) points out that care is a practice and a disposition and both must be present. Struhkamp (2005) and Mol (2006), consider caring primarily as a practice which, in our view, is too restricted to the notion of practical and technical competence. Nursing is, indeed, a practical, hands-on job (Tschudin 2003) and caring can be enhanced if one also develops a caring attitude. However, we think that caring work cannot be done without a caring attitude.

It is obvious that autonomy in responsive relationships is a process. We aimed at describing how people themselves exercise it. Respondents had difficulty in telling the process and one participant explained "it just evolved". This implies that autonomy in responsive relationships develops in a silent way. Silent ways might be interactive standards learned in specific relationships or shared expectations and values. They might evolve over time or through implicit precedents. Yet, it is important to gain more insights into the developmental process to be able to support autonomy in responsive relationship from the onset.

Most theories cover some aspects that are important to patients with diabetes to exercise their autonomy in relationships with others. Our findings presented in this study point to the necessity of an inclusive theory, such as Tronto's, in order to do justice to the different social contexts in which autonomy are realized and in which people play an imperative part in the autonomy process. In addition, people with diabetes perceive that autonomy in responsive relationships can only be realized when a caring attitude accompanies a care activity. Finally, autonomy is promoted in a supportive and responsive environment. At the same time, responsibilities are assumed or shared.

We were particularly interested in how chronically ill people experience their autonomy. An overview of theoretical and empirical literature showed that various concepts of autonomy exist and that autonomy in long-term care is difficult to understand (Moser et al. 2007). People with diabetes are a good example for studying autonomy because of the high prevalence, complexity (co-morbidity and secondary long-term complications), large care demand, and the amount of responsibility that is placed on the patient. Most importantly, they remain under treatment for the rest of their lives, which indicates that long-term relationships with nurses and other health professionals are a natural part of their care context.

We developed a substantive theory that we believe is transferable to people with chronic conditions other than diabetes, but it is imperative that our theory supports meaning-in-context (Leininger 1991). Supporting autonomy in responsive relationships also applies to nurses who are employed in the primary care context and who work with chronically ill people. This enables them to develop long-term relationships with people and understand the patients' context, which in turn is important in supporting relational autonomy. Even though relational aspects of autonomy are mostly related to the nursing and caring profession, we think, that medical professionals too can use our findings to support relational autonomy, especially those who have long-standing (and sometimes life-long) relationships with their patients such as family physicians.

\section{Conclusion}

In this study we investigated how older adults with type 2 diabetes realize autonomy in responsive relationships. We found several processes, namely, preserving patterns of concern and interaction, nurturing collaborative responsibilities, and being engaged in trustful and helpful family relationships that influence the dynamic of that specific relationship. The issue of autonomy in responsive relationships is not confined to a specific health care practice but is an aspect of all relationships within the patient's life context.

According to our respondents, realizing autonomy in responsive relationships takes place when patient, nurses, professionals of the health team and family members carry out care activities supported by a relational attitude of care. People with type 2 diabetes can best realize autonomy in responsive relationships when there is an integration of both relational caring traits and care activities.

Theories of care and caring in our overview focus on attitude, dialogue, activity, relationships and life as a whole. The majority of theories tend to centre on one 
aspect of care and caring which is considered to be essential to good care. However, patients with diabetes can best realize autonomy in relationships with others when several essential aspects of care and caring are present in their lives. Therefore, we advocate a comprehensive approach to care and caring.

It is important for nurses (and other professionals involved in diabetes care) to cultivate relational caring attitudes and express them in their nursing to successfully support patient autonomy in their responsive relationship. Nurses sometimes work in an environment that is not conductive to the cultivation of deeper, caring relationships with their patients due to their heavy work load and time constraints. Ideally, nurses should take on the responsibility to request adequate resources and time. Efforts should not remain at the individual or team level but should be extended to the various policy levels. This is not easy to accomplish since it seems at odds with the current policy of 'production in minutes'. Administrators (and policymakers) should put efforts to preserve a caring environment where qualities such as commitment, engagement, attention and warmth for nurses and patients are valued next to economic concerns. We believe that economic concerns can be integrated in a caring environment. A prerequisite is that responsive relationships remain "workable" or "satisfactory" for patients. Well-cared-for patients who are able to exercise autonomy in responsive relationships are a parameter of high-quality care.

Open Access This article is distributed under the terms of the Creative Commons Attribution Noncommercial License which permits any noncommercial use, distribution, and reproduction in any medium, provided the original author(s) and source are credited.

\section{References}

Artinian, B.M. 1998. Grounded theory research: Its value for nursing. Nursing Science Quarterly 11(1): 5-6.

Blumer, H. 1969. Symbolic interactionism. New York: Englewood Cliffs.

Donchin, A. 2000. Autonomy, interdependence, and assisted suicide: Respecting boundaries/crossing lines. Bioethics 14(3): 187-204.

Friedemann, M.-L. 1989. The concept of family nursing. Journal of Advanced Nursing 14: 211-216.

Friedemann, M.-L., and C. Köhlen. 2003. Familien- und umweltbezogene Pflege. Bern: Hans Huber Verlag.

Gastmans, C. 1999. Care as a moral attitude in nursing. Nursing Ethics 6(3): 214-223.

Gilligan, C. 1982. In a different voice: Psychological theory and women's development. Massachusetts: Harvard University Press.

Hagerty, B.M., and K.L. Patusky. 2003. Reconceptualizing the nursepatient relationship. Journal of Nusing Scholarship 35: 145-150.

Hardwig, J. 1990. What about the family? Hastings Center Report March-April, 5-10.

Ingadottir, B., and S. Halldorsdottir. 2008. To discipline a "dog": The essential structure of mastering diabetes. Qualitative Health Research 18(5): 606-619.
Krouse, H.J., and S.J. Roberts. 1989. Nurse-patient interactive styles: Power, control, and satisfaction. Western Journal of Nursing Research 11(6): 717-725.

Kuczewski, M. G. 1996. Reconceiving the family: The process of consent in medical decisionmaking. Hastings Center Report March-April, 30-37.

Leininger, M.M. 1991. Culture care diversity \& universality: A theory of nursing. New York, NY: National League for Nursing Press.

Mamhidir, A.-G., and B. Lundman. 2004. Having control over type 2 diabetes means daring to be free. Journal of Diabetes Nursing 8: $12-16$.

Mol, A. 2006. De logica van het zorgen: actieve patienten en de grenzen van het kiezen. Amsterdam: Van Gennep.

Morse, J.M., S.M. Solberg, W.L. Neander, J.L. Bottorff, and J.L. Johnson. 1990. Concepts of caring and caring as a concept. Advances in Nursing Science 13(1): 1-14.

Moser, A., H. Van der Bruggen, and G. Widdershoven. 2006. Competency in shaping one's life: Autonomy of people with type 2 diabetes mellitus in a nurse-led, share-care setting; a qualitative study. International Journal of Nursing Studies 43: $417-427$.

Moser, A., R. Houtepen, and G. Widdershoven. 2007. Patient autonomy in nurse-led shared care: A review of theoretical and empirical literature. Journal of Advanced Nursing 57(4): 357-365.

Moser, A., H. Van der Bruggen, G. Widdershoven, and C. Spreeuwenberg. 2008a. Autonomy through identification: A qualitative study of the process of identification used by people with type 2 diabetes. Journal of Clinical Nursing 17(7b): 209-216.

Moser, A., H. Van der Bruggen, G. Widdershoven, and C. Spreeuwenberg. 2008b. Self-management of type 2 diabetes mellitus: a qualitative investigation from the perspective of participants in a nurse-led, shared-care programme in the Netherlands. BMC Public Health 8: 91.

Moser, A., R. Houtepen, H. Van der Bruggen, C. Spreeuwenberg, and G. Widdershoven. 2009. Autonomous decision making and moral capacities. Nursing Ethics 16(2): 203-218.

Nagelkerk, J., K. Reick, and L. Meengs. 2006. Perceived barriers and effective strategies to diabetes self-management. Journal of Advanced Nursing 54(2): 151-158.

Noddings, N. 1984. Caring-a feminine approach to ethics and moral education. Berkeley, CA: University of California Press.

Parker, R.S. 1990. Nurses' story: the search for a relational ethics of care. Advances in Nursing Science 13(1): 31-40.

Paterson, B.L., and J. Sloan. 1994. A phenomenological study of the decision-making experience of individuals with longstanding diabetes. Canadian Journal of Diabetes Care 18(4): 10-19.

Paterson, B., S. Thorne, and M. Dewis. 1998. Adapting to and managing diabetes. Image: Journal of Nursing Scholarship 30(1): 57-62.

Peplau, H. 1988. Interpersonal relations in nursing. Hampshire and London: Macmillan Education Ltd.

Roberts, S.J., H.J. Krouse, and P. Michaud. 1995. Negotiated and nonnegotiated nurse-patient interactions. Clinical Nursing Research 4(1): 67-77.

Strauss, A., and J. Corbin. 1990. Basics of qualitative research: Grounded theory procedures and techniques. Newbury Park: Sage.

Struhkamp, R.M. 2005. Patient autonomy: A view from the kitchen. Medicine, Health Care and Philosophy 8: 105-114.

Tarlier, D.S. 2004. Beyond caring: The moral and ethical bases of responsive nurse-patient relationship. Nursing Philosophy 5: 230-241.

Tronto, J.C. 1993. Moral boundaries: A political argument of an ethic of care. New York: Routledge.

Tschudin, V. 2003. Ethics in nursing: The caring relationship, 3rd ed. London: Butterworth Heinemann. 
Verkerk, M. 2001. The care perspective and autonomy. Medicine, Health Care and Philosophy 4: 289-294.

Watson, J. 1988. Nursing: Human science and human care. A theory of nursing. New York: National League for Nursing.

Widdershoven, G. 1999. Care, cure and interpersonal understanding. Journal of Advanced Nursing 29(5): 1163-1169.
Williams, G.H., and P.H.N. Wood. 1988. Coming to terms with chronic illness: The negotiation of autonomy in rheumatoid arthritis. International Journal of Disability Studies 10(3): 128133. 